\title{
Evidence of Immune Genes in the Crinoid: Antedon Bifida Evidence of A. Bifida Igkappa Gene, Fc Receptor Gene
}

\author{
Michel Leclerc ${ }^{1 *}$, Franck Letourneur, Dominique Davoult, Ariane Jolly and Pierre de la Grange \\ ${ }^{1}$ Immunology of Invertebrates University of Orleans, France
}

Received: June 27, 2018; Accepted: July 10, 2018; Published: July 12, 2018

*Corresponding authors: Michel Leclerc, Immunology of Invertebrates University of Orleans, 556 rue Isabelle Romée, 45640 Sandillon, France, Email: mleclerc45@gmail.com

\section{Abstract \\ Immuno-genomics studies realized in Echinodermata (Invertebrates) were surprising. 3 classes out of 5 Echinodermata presented an IGKappa gene and an Fc receptor gene. It was, first demonstrated, in Asterids and Ophuirids. It was, secondly clearly shown, in the ancestral Crinoïd: Antedon bifida.}

Keywords: Invertebrates; Echinodermata; Crinoids; Igkappa Gene; Fc Gene; A. Bifida;

\section{Introduction}

The general idea that emerged from the experiments, made in our laboratory, was that Echinodermata, as exemplified, by sea star Asterias rubens (Asterids)and by Ophiocomina nigra (Ophuirids), possessed an immune system, able to mount cellular and humoral-specific responses,

After stimulation with a foreign antigen: the horse-radish peroxides (HRP) [1, 2]. Then, these echinodermata produced a primitive antibody, correlated to an Igkappa gene $[3,4]$ to a Fab gene [5], an Fc receptor gene [6].But a question deserves to be put: did the ancestral Echinodermata, Antedon bifida, (Crinoïd) possess such genes? It is why, in a first time, we look for IgKappa gene, Fab gene, Fc receptor gene in these crinoids by the mean of genomic studies.

\section{Materials and Methods}

\section{Animals}

Antedon bifida was obtained at the station « Of Biologie Marine of Roscoff » France.

\section{Obtention of crinoids mRNA}

Digestive coeca were excised from the A. bifida body.

Bifida mRNA was obtained from Uptizol (Interchim). Quality control was operated.

\section{Sequencing}

Sequencing was made on Illumina Next Seq 500 with paired-end: 2.75 bp Transcriptome was assembled from RNASeq fastq files using Trinity v2.1.1 [7] with default parameters. A BLAST database was created with the assembled transcripts using makeblastdb application from ncbi-blast+ (v2.2.31+). The sequences of transcripts of interest were then blasted against this database using blastn application from ncbi-blast+ [8] with parameter word size 7 .

\section{Results}

The (Table 1) which is following summarizes the found Antedon bifida transcriptomes of Igkappa gene and Fc receptor gene of IgA (FCAR) of IgE (Fcer2a) we met in Homo sapiens and in Mus musculus:

\begin{tabular}{|c|c|c|c|c|c|c|c|c|c|}
\hline Query ID & $\begin{array}{l}\text { Query } \\
\text { Symbol }\end{array}$ & Species & Subject ID & $\begin{array}{l}\text { Identity } \\
\text { (\%) }\end{array}$ & Length & Mis-match & $\begin{array}{c}\text { Gap } \\
\text { open }\end{array}$ & $\begin{array}{c}\text { Query } \\
\text { cover (\%) }\end{array}$ & E-value \\
\hline ВС032451.1 & Igk & $\begin{array}{l}\text { Homo } \\
\text { sapiens }\end{array}$ & $\begin{array}{c}\text { TRINITY_DN9178_ } \\
\text { c0_g1_i2 }\end{array}$ & 89,74 & 78 & 7 & 1 & 8 & $4,00 \mathrm{E}-20$ \\
\hline NM_133273.3 & FCAR & $\begin{array}{l}\text { Homo } \\
\text { sapiens }\end{array}$ & $\begin{array}{c}\text { TRINITY_DN13535_ } \\
\text { c0_g1_i1 }\end{array}$ & 79,31 & 87 & 13 & 5 & 4 & $5,00 \mathrm{E}-07$ \\
\hline NM_001253737.1 & Fcer2a & $\begin{array}{l}\text { Mus } \\
\text { musculus }\end{array}$ & $\begin{array}{c}\text { TRINITY_DN20232_ } \\
\text { c5_g2_i1 }\end{array}$ & 84,31 & 51 & 7 & 1 & 2 & $8,00 \mathrm{E}-05$ \\
\hline
\end{tabular}




\section{The Antedon bifida IgK transcriptome sequence is the one}

>TRINITY_DN9178_c0_g1_i2(Igk)

5'AGCGAATGAAAAAGAAGAACCGGCCAAAAAAAGTACTTCTACCAAAGAAGCGAATGAAAAAGAAGAACCGGCCAAAAAAAGTACTTCTACCAAAGA AGAAACTGAAATAGAAGAACTAACCGAAACAAGTATTTCTACAAAATCAGTTTCTGCCAGTGATATATTCCTTGGTACAACTTTCACACTGGAGATGGG ATTCTGCGTAGGACCTGAACACAAACCGTTTACAGGAGATTTCGACGGTGACGGTAATGAAGATCTTCTGTTTCACAATTCAAAGACAGGCTCGAAAA AGATATACTATGCAAGTTGTGACGGCTCTTTTAATGGTGATAGGTCGTGGAGAAGAGAGATGAATTTTTGCTACGTAAGTGGATATGATCTATACATTG GTGATTTCAACGGCGATGGTCGATCCGATATGCTGTGTCATCGTCCTCAGTATGGTCAGATTTGGGTTGTGTTGGCGCAACCTGGGGGTGTATTCACTG CTAACCCGTGGTCGTATAGTCCCAATTGGTGCAAGGCCACCACTGATAAAGTATATATTGGAGACTTCAACGCAGACGGTCGGGATGATATTCTTTGCCA CACACAAAGTTCGGGTTACATTGCAATATATTATGCATTATACACTGGTTATTTTTCTACCTCTACAACATATCGCTTTACACGAAGTATGAGTTGGTGC AGAGGTACATATCAAAGAGTGTATACTGGAGATTTCAACGGAGACCGAAGGGTTGATATGCTCTGCCACGACTACTCATCTGGCTACATATATGTAGCAG TAGCCACAGCGACTGGTGGATTCACCTCTGCCACATGGAGCAGAAGTATGGGCTGGTGCAAGCATTCGAACTCTAAGCTCAGCATTGGAGATTTCAATA AAGATAACCGCGACGACATCATGTGCAGCGACACAAATGGTCCTTACTGGATAGCATTCTCTCTGTACAACGGTTCGTTTTCATCTAAAAGCTGGACCC GTAAACAAAACTGGTGTACATCTGGCAATGATGTGTTAGTTTCGGATGTGAATGGAGATGGTGGGGATGATTTGATGTGCCATAATGAAGCCGACGGCA TCAAGTACATATCGATCAACCATAAGGCCTAAAGCAAGTTCCTCTCAATATTACAGAAAACTATTCACCACAAATGATTCATTTTGTACTGAACCTCAAT TCAAATTCAATTAAAATTTACATAAACGTTAACGGAAGGATACAATCAACTAAAATAATGTTCATTCATTATTTTTCGTCGATAACCTAAACAAAAATC AGATAAGAAATTATACAATAATATACTGTAAACGTATTATACAAAATAATTAAATGTATATTAAGCTACTGTACTTAGAAATGTACTTGTACGCTTATTAA TATTAATAAGCCTAATGCCCGGGTTGATAATAATAAAATACATTTTTGCAAGTTCAAAAAAAAAAAAAAAAAAAAAAAAAAAAAAAAAAAAAAAAAA AAAAAAAACTCAAAAGTCCCAGGCCCCACCCCGACCTACTGAACCAGAAAG3'

The Antedon bifida Fc receptor (FCAR) transcriptome sequence is the one: $>$ TRINITY_DN13535_c0_g1_i1(FCAR)

5'ACTCTACGAACCAAATTTAAATATAACCCGAGAATGTATGTACAACCGATCCAGTAAAATGGTAACATTCAAGACTACGATTATGTACCTTGTTAAATT AAAATAAAATAAAGTACCGGTAGATACACCATCATAAATATCAGCCTTTCATCAAGCAAAACAGTCATTTGTTTACAGTTCTGTCTTGAGTAGATGTTC TCAGCAAATTTTTCTAATATAACAATTATAAACTTCTTGTGAAGATGAAATTTATTGATTTAATTGGATGCCAGTTAATTTTATTTAGAATTATTTTTA ATTTTGATCTGAGAAACAGAATTAAGAACTGGAAAAGAAATGAACAAAAGTTTCCATAAAACTATCGTTATTCAATTTAGTTTGTTTCTATACAATGG CAAATTAACACTTTCAAGAGAGGTGCATATTTTTATTTTTGGTCAACTATGCTTACAATAGGTAAAATAAAATATTAACATCCCCCTACCCTACTTACAT CCCAACTTATCACGTAATAAACCTTATTTCTCTGTCGGGAAAATTTCAGATTTGAGCAAGTATAATTTTATTTATTATTTCAGAATGTTCTCTATTTTAA CTGGGTGCTTGATACAATTAACATTGTAGAAGTTTATTTGTTTATTTG TTTATTTGTTTGTTTGTTTGTTTGTTTAGATGGAGTTTCTCTCTTGTTGTC

\section{CAGGCTGGAGTGCAATGGCA3'}

\section{Discussion and Conclusion}

Fortunaly, we find again in another Echinodermata, the IgKappa gene, the Fc receptor gene, we found in the Asterid: Asterias Rubens and in the Ophuirid: Ophiocomina nigra. Fab gene was found also in A.bifida, but the e-value was not significant. A considerable role may be assigned to crinoids IgKappa gene and Fc receptor gene: it is the one of Antedon bifida primitive antibody, but further research is needed in order, to examine and characterize the "immunocytochemical aspects of immunizations" to HRP (Horse-radish peroxides) or other antigen in Antedon bifida.

Nevertheless, for the third time, we find an invertebrate primitive antibody in an Echinodermata: it's a great novelty!

\section{References}

1. Leclerc M. Ultrastractural study of the reactions of asterina gilbbosa (Echinoderm Asteride) at the level of the axial organ after injection of proteins. Ann. Immunol. 1973;124:363-374.

2. Leclerc M. Immunocyto chemical Reactions in the Brittle-Star: Ophiocomina Nigra after Immunization. Et al 2017, Int.J. Vaccines and Vaccine. 2017;4(4):00088

3. Leclerc M. A True New Gene: An Invertebrate Antibody Igkappa Gene. Amer.J.Immunol. 2013;9(4):101-102.

4. Leclerc M. Adaptative Immunity, a True New Gene in Ophiocomina Nigra: An Ophuirid Igkappa Gene. Cell. Cellular. Life. Sci.J. 2018;3(1):000117

5. Leclerc M. Evidence of Fab Fragment Gene in an Invertebrate: The Sea Star Asterias Rubens. EC Microbiology. 2016;3(5):539-541.

6. Leclerc, M. Evidence of CR Receptor Gene in an Invertebrate. EC Microbiology. 2016;4(5):759-760.

7. Grabher MG. Full-length transcriptome assembly from RNASeq data without a reference genome. Nature Biotechnology. 2011;29(7):644-652.

8. Altschul SF. Basic local alignment search tool. J.Mol.Biol. 1990;215(3):403-410. 